\title{
Tandem ROMP-Hydrogenation with a Third-Generation Grubbs Catalyst
}

\author{
Kenneth D. Camm, Nemesio Martinez Castro, Yuwen Liu, Pawel Czechura, Jennifer L. \\ Snelgrove and Deryn E. Fogg* \\ Center for Catalysis Research and Innovation, Department of Chemistry, University of Ottawa, \\ 10 Marie Curie, Ottawa ON, Canada, K1N 6N5
}

\section{SUPPORTING INFORMATION}

\section{Contents:}

1. Composite Reaction Profiles for Tandem Hydrogenation of All Monomers.

2. Experimental Procedures.

3. ${ }^{1} \mathrm{H}$ NMR Spectra.

4. References.

*Corresponding Author, E-mail: dfogg@uottawa.ca. Fax: (613) 562-5170 
1. Composite Reaction Profiles for Tandem Hydrogenation of All Monomers. [N.B. Colour and symbol scheme differ from MS.]

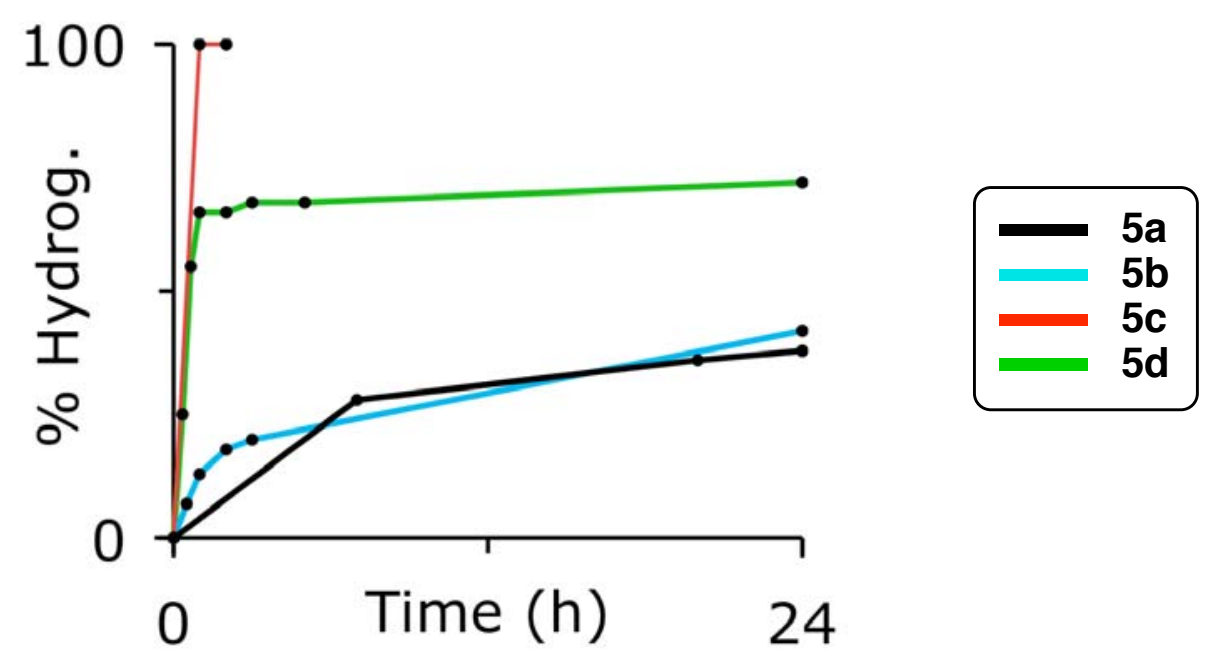

Figure S1. Tandem hydrogenation of all monomers (5a-d) using $\mathbf{3}$ as catalyst: standard procedure, without added $\mathrm{PCy}_{3}$.

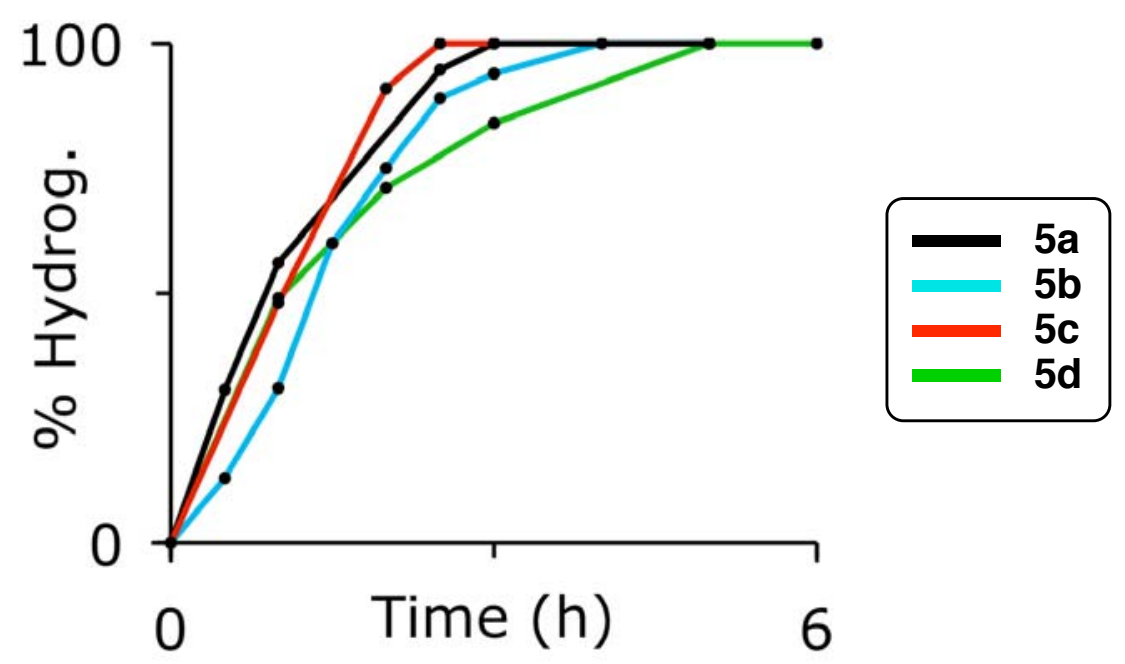

Figure S2. Tandem hydrogenation of all monomers (5a-d) using $\mathbf{3}$ as catalyst: procedure as in Figure S1, but with 1.2 equiv $\mathrm{PCy}_{3}$ added post-ROMP. 


\section{Experimental Procedures.}

General Procedures. All reactions were carried out at room temperature $\left(22^{\circ} \mathrm{C}\right)$ under $\mathrm{N}_{2}$ or argon, using standard Schlenk or drybox techniques. Dry, oxygen-free solvents were obtained using an Anhydrous Engineering solvent purification system, and stored over Linde $4 \AA$ molecular sieves in a drybox. Triethylamine, methanol and $\mathrm{CDCl}_{3}$ were distilled from $\mathrm{CaH}_{2}$, degassed by consecutive freeze/pump/thaw cycles, and stored over Linde $4 \AA$ molecular sieves. ${ }^{1} \mathrm{H}$ NMR (300 or $500 \mathrm{MHz}$ ) spectra were recorded on a Bruker Avance-300 or Bruker AMX-500 spectrometer. GPC data were measured using $\mathrm{CH}_{2} \mathrm{Cl}_{2}$ as eluent (flow rate $1.0 \mathrm{~mL} / \mathrm{min}$; sample 1 $\mathrm{mg} / \mathrm{mL}$ ) on a Wyatt Technology DAWN light-scattering GPC instrument equipped with an Optilab DSP refractometer, an HPLC system with a Waters model 515 HPLC pump, Rheodyne model 7725i injector with $200 \mu \mathrm{L}$ injection loop, and Waters Styragel HR3 and HR4 columns in series. IR spectra were recorded on a Bomem MB100 spectrometer. Complexes $\mathbf{1},{ }^{1} \mathbf{2},{ }^{2,3} \mathbf{3},{ }^{4,5}$ and monomers $\mathbf{5} \mathbf{a}^{6}, \mathbf{5} \mathbf{b},{ }^{7}$ and $\mathbf{5} \mathbf{d}^{8}$ were prepared according to literature procedures. Organic reagents were purchased from Aldrich and used without further purification. Ethylvinyl ether was degassed by consecutive freeze/pump/thaw cycles. Hydrogen (UHP Grade) was purchased from BOC Gases and used without further purification.

Preparation of (N-benzyl)-5-norbornene-endo-2,3-dicarboximide (5c). Monomer 5c was prepared by minor modification of the method of Khosravi et al. ${ }^{9,10}$ A mixture of 5-norborneneendo-2,3-dicarboxylic anhydride $(1.00 \mathrm{~g}, 6.09 \mathrm{mmol})$, benzylamine $(0.653 \mathrm{~g}, 6.09 \mathrm{mmol}, 0.665$ $\mathrm{mL})$ and triethylamine $(0.678 \mathrm{~g}, 6.70 \mathrm{mmol}, 0.934 \mathrm{~mL})$ in toluene $(40 \mathrm{~mL})$ was refluxed for $19 \mathrm{~h}$ until it formed a homogeneous solution. The solution was allowed to cool and washed with $10 \%$ aq. $\mathrm{HCl}(3 \times 20 \mathrm{~mL})$, brine, then dried $\left(\mathrm{MgSO}_{4}\right)$, filtered, and concentrated to yield a white solid. 
Yield: $1.44 \mathrm{~g}, 93 \% .{ }^{1} \mathrm{H}\left(300 \mathrm{MHz}, \mathrm{CDCl}_{3}\right): \delta 7.27(\mathrm{~m}, 5 \mathrm{H}, \mathrm{Ph}), 5.91(\mathrm{~m}, 2 \mathrm{H}, \mathrm{HC}=\mathrm{CH}) ; 4.50(\mathrm{~s}$, $\left.2 \mathrm{H}, \mathrm{N}-\mathrm{CH}_{2} \mathrm{Ph}\right), 3.37(\mathrm{~m}, 2 \mathrm{H}, \mathrm{CH}-\mathrm{CH}-\mathrm{CH}), 3.28(\mathrm{~m}, 2 \mathrm{H}, \mathrm{CHCO}), 1.72\left(\mathrm{dd},{ }^{3} \mathrm{~J}_{\mathrm{HH}}=1.6,8.8 \mathrm{~Hz}\right.$, $\left.1 \mathrm{H}, \mathrm{CH}-\mathrm{CH}_{2}-\mathrm{CH}\right), 1.54\left(\mathrm{dd},{ }^{3} J_{\mathrm{HH}}=1.6,8.8 \mathrm{~Hz}, 1 \mathrm{H}, \mathrm{CH}-\mathrm{CH}_{2}-\mathrm{CH}\right)$. IR (Nujol): v(CO) $1765 \mathrm{~cm}^{-1}$. HR-MS (+EI): Calc'd for $\mathrm{C}_{16} \mathrm{H}_{15} \mathrm{O}_{2} \mathrm{~N}\left[\mathrm{M}^{+}\right], m / z$ 253.11028. Found, $m / z$ 253.11426.

\section{Representative ROMP and Tandem ROMP-Hydrogenation Procedures.}

(a) ROMP of bis(1,2:3,4-di-O-isopropylidene- $\alpha$-galactopyranos-6-O-yl)-5-norbornenetrans-2,3-dicarboxylate (5a). A solution of $3(2.6 \mathrm{mg}, 3.6 \mu \mathrm{mol})$ in $260 \mu \mathrm{L} \mathrm{CH}_{2} \mathrm{Cl}_{2}$ was added in one portion to a rapidly-stirred solution of $\mathbf{5 a}(121 \mathrm{mg}, 0.18 \mathrm{mmol})$ in $3 \mathrm{~mL} \mathrm{CH}_{2} \mathrm{Cl}_{2}$. Conversions were determined by ${ }^{1} \mathrm{H}$ NMR analysis, by monitoring the decrease in the olefinic resonances due to monomer $(6.27-6.07 \mathrm{ppm})$ relative to the galactopyranose $\mathrm{C}-5$ multiplet at $4.59 \mathrm{ppm}$. After complete conversion, the polymerization was quenched by adding ethylvinyl ether, and stirred for 20 minutes. Removal of volatiles and extraction with hexanes afforded polymer in quantitative yield. Subsequent purification was undertaken by dissolving the polymer in the minimum volume of $\mathrm{CH}_{2} \mathrm{Cl}_{2}$ and adding the solution dropwise to vigorously-stirred, icecold methanol $(20 \mathrm{~mL})$ : yields $>90 \%$. NMR data agree with values previously reported. ${ }^{6}$

(b) Tandem ROMP-Hydrogenation of [5a $]_{50}$. A solution of $2(3.0 \mathrm{mg}, 3.6 \mu \mathrm{mol})$ in 300 $\mu \mathrm{L} \mathrm{CH} \mathrm{Cl}_{2}$ was added in one portion to a rapidly-stirred solution of $\mathbf{5 a}(121 \mathrm{mg}, 0.18 \mathrm{mmol})$ in 3 $\mathrm{mL} \mathrm{CH}_{2} \mathrm{Cl}_{2}$. Once reaction was complete, the solution was diluted with $13.6 \mathrm{~mL} \mathrm{CH}_{2} \mathrm{Cl}_{2}$, and $\mathrm{NEt}_{3}(5 \mu \mathrm{L}, 0.036 \mathrm{mmol})$ was added, then $3.4 \mathrm{~mL} \mathrm{MeOH}$, affording a final volume of $20 \mathrm{~mL}$. The solution was purged with $\mathrm{H}_{2}$ in a glass-lined autoclave, pressurized to $1000 \mathrm{psi}$ and stirred at $60{ }^{\circ} \mathrm{C}$. To establish time profiles, samples were removed at intervals for ${ }^{1} \mathrm{H}$ NMR analysis, and the decrease in integrated intensity of the olefinic peaks $(5.60-5.30 \mathrm{ppm})$ relative to the 
galactopyranose C-5 multiplet at $4.59 \mathrm{ppm}$ was measured. Once hydrogenation was complete, the polymer was isolated in quantitative yield, as described in (a). ${ }^{1} \mathrm{H} \mathrm{NMR}\left(\mathrm{CDCl}_{3}, 300 \mathrm{MHz}\right)$ : $\delta 5.48$ (br s, 2H, galactopyranose $\mathrm{C}-1 \mathrm{CH}$ ), 4.59 (br m, 2H, galactopyranose $\mathrm{C}-5 \mathrm{CH}$ ), 4.28-4.13 (br m, 8H, galactopyranose $\mathrm{CH}+\mathrm{CH}_{2}$ ), $3.98($ br m, 2H, galactopyranose $\mathrm{CH}$ ), $3.13($ br m, $1 \mathrm{H}$, cyclopentane $\mathrm{CHCO}$ ), 2.78 (br m, 1H, cyclopentane $\mathrm{CHCO}$ ), 2.2-1.8 (br m, 2H, cyclopentane $\mathrm{CH}$ ), 1.8-1.5 (br m, 2H, cyclopentane $\mathrm{CH}_{2}$ ), 1.5-1.0 (br m, 4H, polymer backbone $\mathrm{CH}_{2}$ ); 1.46 (s, 6H, acetal $\mathrm{CH}_{3}$ ), $1.41\left(\mathrm{~s}, 6 \mathrm{H}\right.$, acetal $\left.\mathrm{CH}_{3}\right), 1.30$ (br s, 12H, acetal $\mathrm{CH}_{3}$ ). IR (Nujol): $v(\mathrm{CO}) 1730$ $\mathrm{cm}^{-1}$.

(c) PCy $y_{3}$-Assisted Tandem ROMP-Hydrogenation of $[\mathbf{5 a}]_{50}$. The reaction was carried out as above, using initiator $3(2.6 \mathrm{mg}, 3.6 \mu \mathrm{mol})$, with addition of $\mathrm{PCy}_{3}(1.2 \mathrm{mg}, 4.3 \mu \mathrm{mol})$ after ROMP was complete, and 5 min prior to addition of $\mathrm{NEt}_{3}$.

(d) ROMP of (N-hydroxysuccinimide)-5-norbornene-2-carboxylate (5d). This procedure was carried out as described in (a), but at $-20{ }^{\circ} \mathrm{C}$. Conversions were determined by ${ }^{1} \mathrm{H}$ NMR analysis, by monitoring the decrease in olefinic resonances due to monomer $(6.26-6.06 \mathrm{ppm})$ relative to the increase in those due to polymer (5.68-5.25 ppm). After complete conversion, the polymer was isolated and purified as above. NMR data agree with values previously reported. ${ }^{8}$

\section{Polymer Characterization Data.}

(a) ROMP of $5 \boldsymbol{b} .{ }^{1} \mathrm{H}$ NMR $\left(\mathrm{CDCl}_{3}, 300 \mathrm{MHz}\right): \delta 5.57-5.25$ (br m, 2H, polymer backbone $\mathrm{CH}$ ), 4.62 (br m, 2H, menthol $\mathrm{CHO}$ ), 3.51-2.69 (br m, 4H, cyclopentane $\mathrm{CH}$ ), 2.05-1.15 (br m, 19H, menthol $\mathrm{CH}$, menthol $\mathrm{CH}_{2},{ }^{i} \mathrm{Pr} \mathrm{CH}$, cyclopentane $\mathrm{CH}_{2}$ ), 1.05-0.6 (br m, 19H, cyclopentane $\mathrm{CH}_{2}$, menthol $\mathrm{CH}_{3},{ }^{i} \mathrm{Pr} \mathrm{CH}_{3}$ ). IR (Nujol): v(CO) $1729 \mathrm{~cm}^{-1}$. 
(b) $P C y_{3}$-Assisted Tandem ROMP-Hydrogenation of $5 \boldsymbol{b} .{ }^{1} \mathrm{H}$ NMR $\left(\mathrm{CDCl}_{3}, 300 \mathrm{MHz}\right): \delta$ 4.65 (br m, 2H, menthol $\mathrm{CHO}$ ), 3.16-2.72 (br m, 2H, cyclopentane $\mathrm{CHCO}$ ), 2.30-0.55 (br m, 44H, cyclopentane $\mathrm{CH}$, cyclopentane $\mathrm{CH}_{2}$ menthol $\mathrm{CH}$, menthol $\mathrm{CH}_{2}$, menthol $\mathrm{CH}_{3},{ }^{i} \mathrm{Pr} \mathrm{CH},{ }^{i} \mathrm{Pr}$ $\mathrm{CH}_{3}$, polymer backbone $\mathrm{CH}_{2}$ ). IR (Nujol): $v(\mathrm{CO}) 1728 \mathrm{~cm}^{-1}$.

(c) ROMP of $5 \boldsymbol{c} .{ }^{1} \mathrm{H}$ NMR $\left(\mathrm{CDCl}_{3}, 300 \mathrm{MHz}\right): \delta 7.27$ (br m, 5H, Ph), 5.62 (br m, 2H, polymer olefinic backbone $\mathrm{CH}$ ), 4.60 (br m, $2 \mathrm{H}, \mathrm{N}-\mathrm{CH}_{2} \mathrm{Ph}$ ), 3.40-2.60 (br m, 4H, cyclopentane $\mathrm{CH}$ ), 2.05-0.8 (br m, 2H, cyclopentane $\mathrm{CH}_{2}$ ). IR (Nujol): $v(\mathrm{CO}) 1770 \mathrm{~cm}^{-1}$.

(d) Tandem ROMP-Hydrogenation of $\mathbf{5 c} .{ }^{1} \mathrm{H} \mathrm{NMR}\left(\mathrm{CDCl}_{3}, 300 \mathrm{MHz}\right) \delta 7.27$ (br m, 5H, $\mathrm{Ph}$ ), $4.60\left(\mathrm{~s}, 2 \mathrm{H}, \mathrm{N}-\mathrm{CH}_{2} \mathrm{Ph}\right), 3.14$ (br m, 2H, cyclopentane $\mathrm{CHCO}$ ), 2.20-0.48 (br m, 8H, cyclopentane $\mathrm{CH}_{2}$, cyclopentane $\mathrm{CH}$, polymer backbone $\mathrm{CH}_{2}$ ). IR (Nujol): $v(\mathrm{CO}) 1769 \mathrm{~cm}^{-1}$.

(e) PCy $y_{3}$-Assisted Tandem ROMP-Hydrogenation of $5 \boldsymbol{d} .{ }^{1} \mathrm{H} \mathrm{NMR}\left(\mathrm{CDCl}_{3}, 300 \mathrm{MHz}\right): \delta$ 3.21 (br m, 1H, cyclopentane CHCO), 2.79 (br m, 4H, N-Succinimide $\mathrm{CH}_{2}$ ), 2.39-1.80 (br m, $4 \mathrm{H}$, cyclopentane $\mathrm{CH}_{2}+\mathrm{CH}$ ); 1.75-1.50 (br m, 2H, cyclopentane $\mathrm{CH}_{2}$ ), 1.50-0.75 (br m, 4H, polymer backbone $\mathrm{CH}_{2}$ ). IR (Nujol): $v(\mathrm{CO}) 1739 \mathrm{~cm}^{-1}$. 


\section{3. ${ }^{1}$ H NMR Spectra}

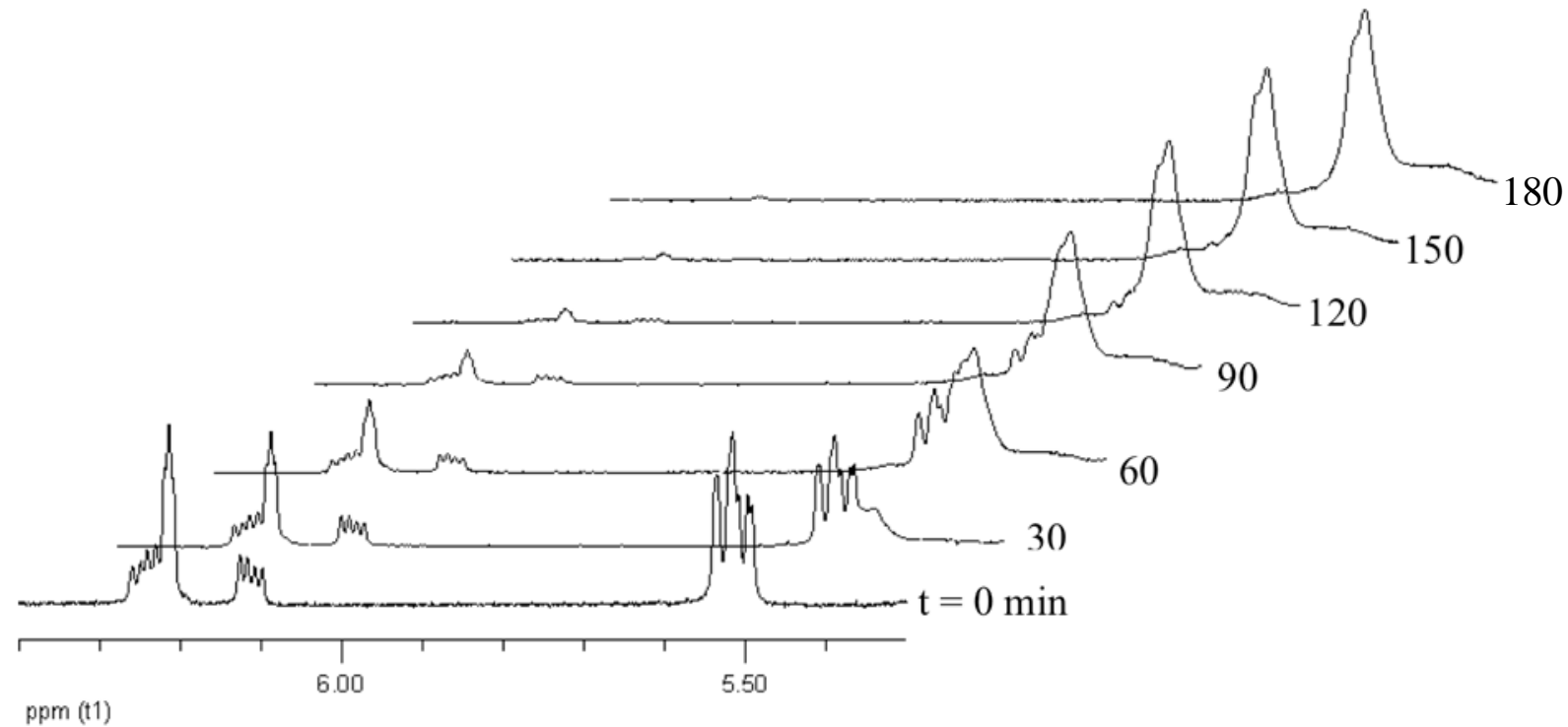

(a) Representative ${ }^{1} \mathrm{H}$ NMR spectrum (300 $\mathrm{MHz}, \mathrm{CDCl}_{3}$ ), showing changes in the olefinic region over the timescale of ROMP of $\mathbf{5 a}$ by initiator $\mathbf{2}$.

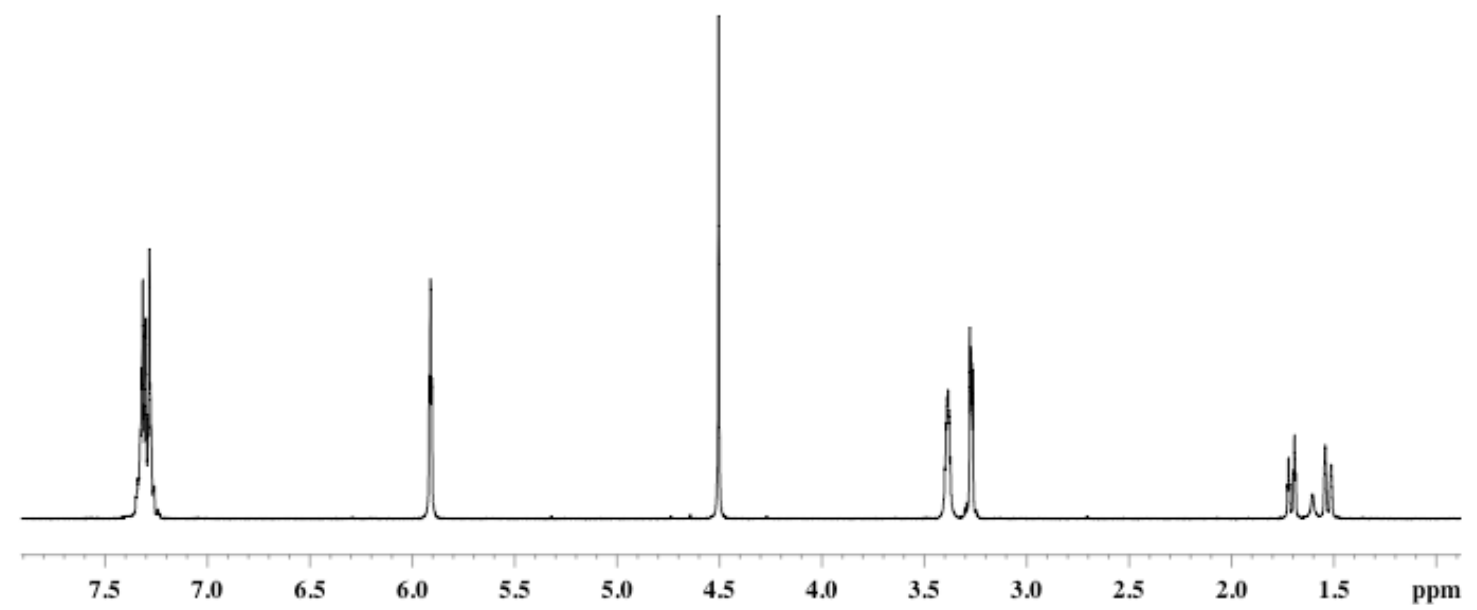

(b) ${ }^{1} \mathrm{H}$ NMR spectrum $\left(300 \mathrm{MHz}, \mathrm{CDCl}_{3}\right)$ of $\mathbf{5 c}$. 


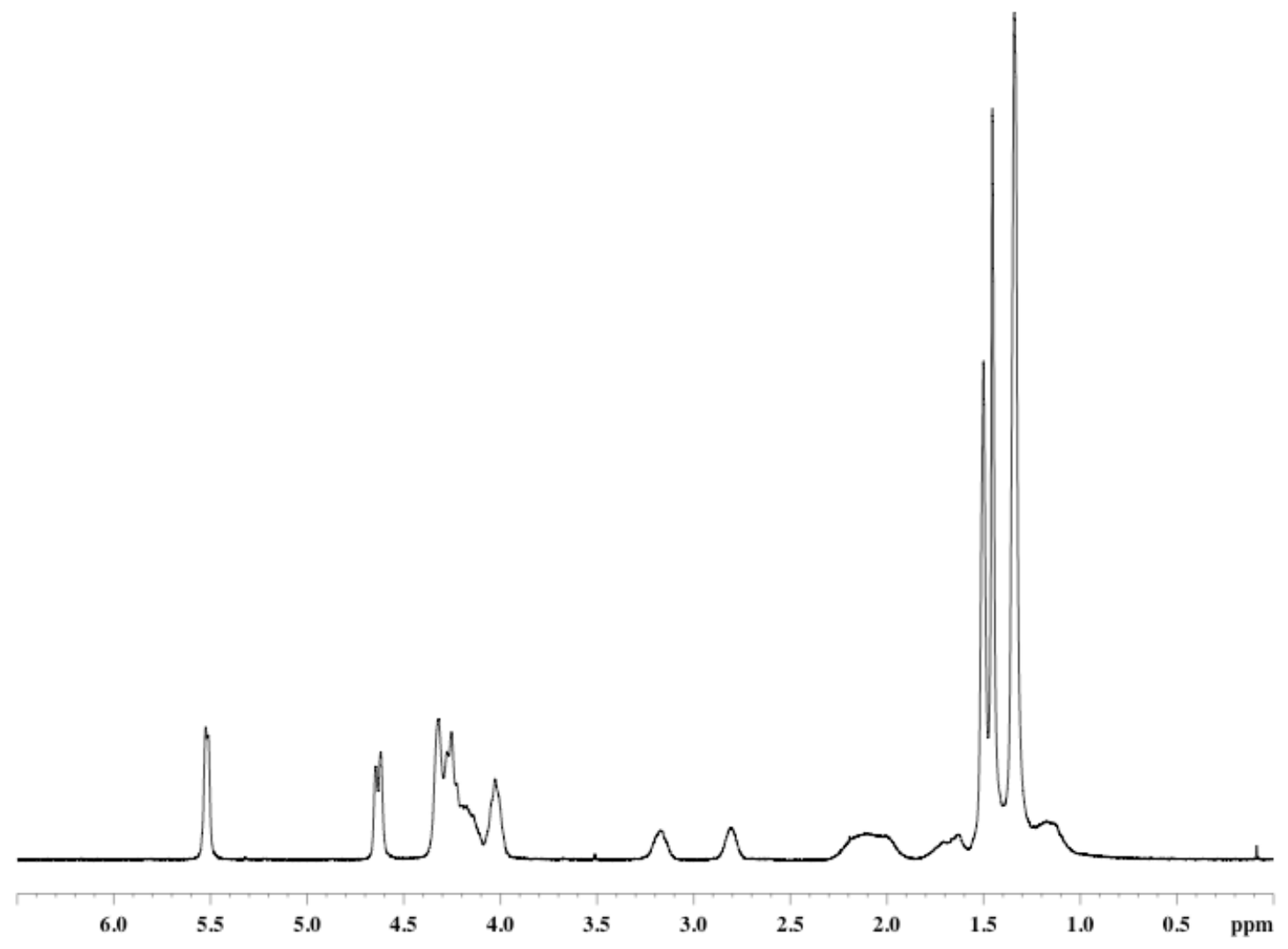

(c) ${ }^{1} \mathrm{H}$ NMR spectrum $\left(300 \mathrm{MHz}, \mathrm{CDCl}_{3}\right)$ of reduced $[\mathbf{5 a}]_{50}$. 


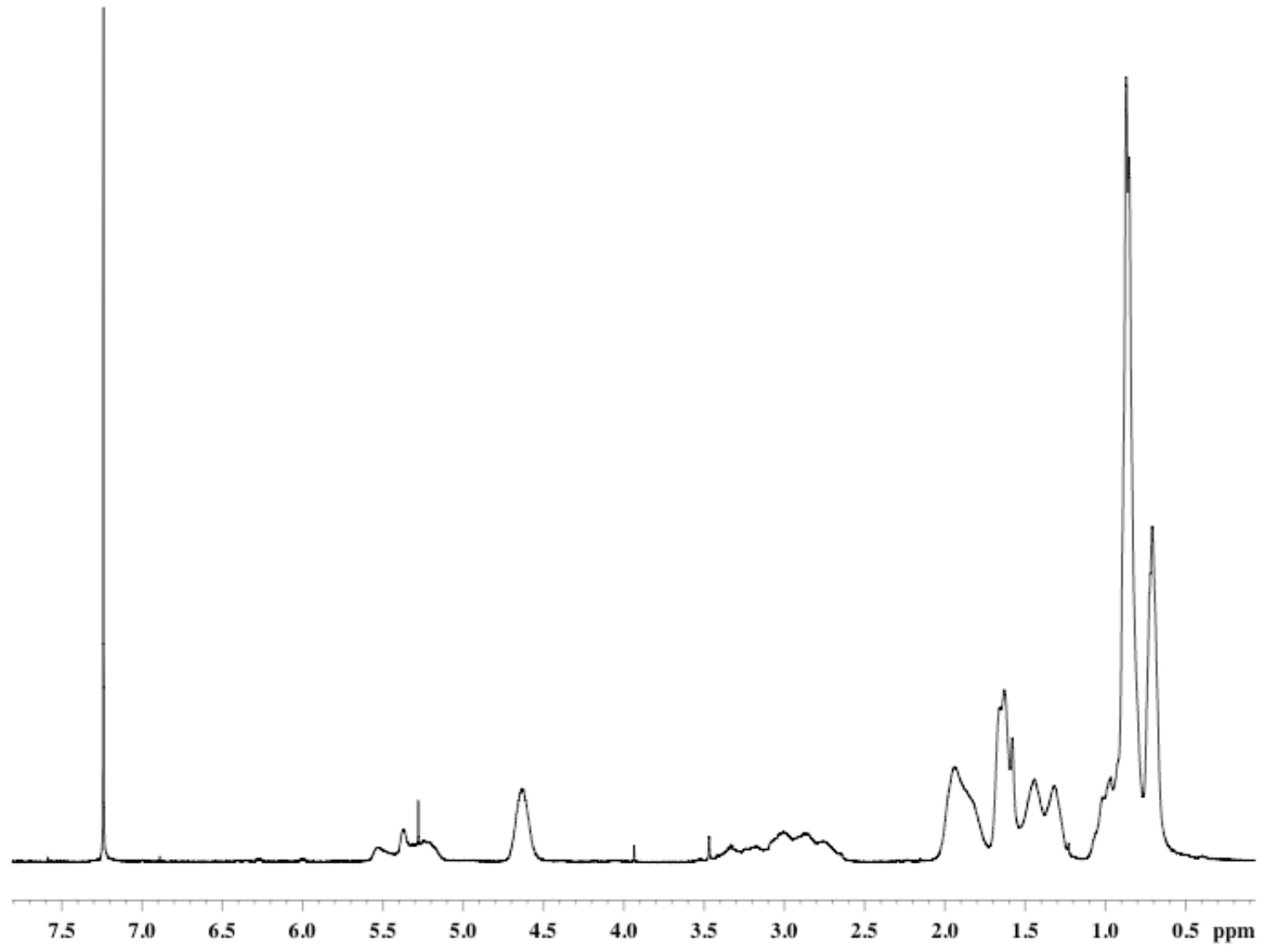

(d) ${ }^{1} \mathrm{H}$ NMR spectrum $\left(300 \mathrm{MHz}, \mathrm{CDCl}_{3}\right)$ of $[\mathbf{5 b}]_{50}$. 


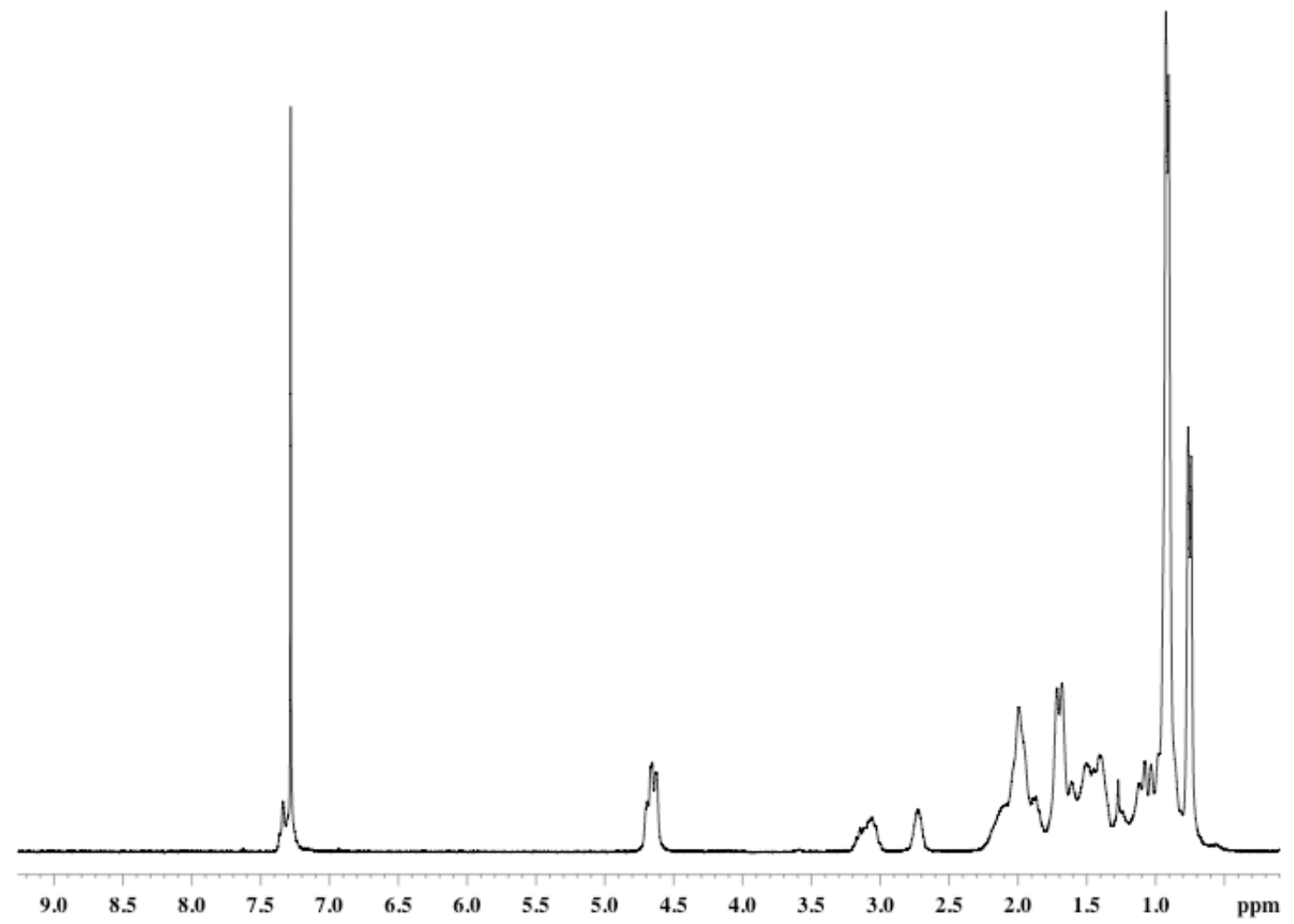

(e) ${ }^{1} \mathrm{H}$ NMR spectrum $\left(300 \mathrm{MHz}, \mathrm{CDCl}_{3}\right)$ of reduced $[\mathbf{5 b}]_{50}$.

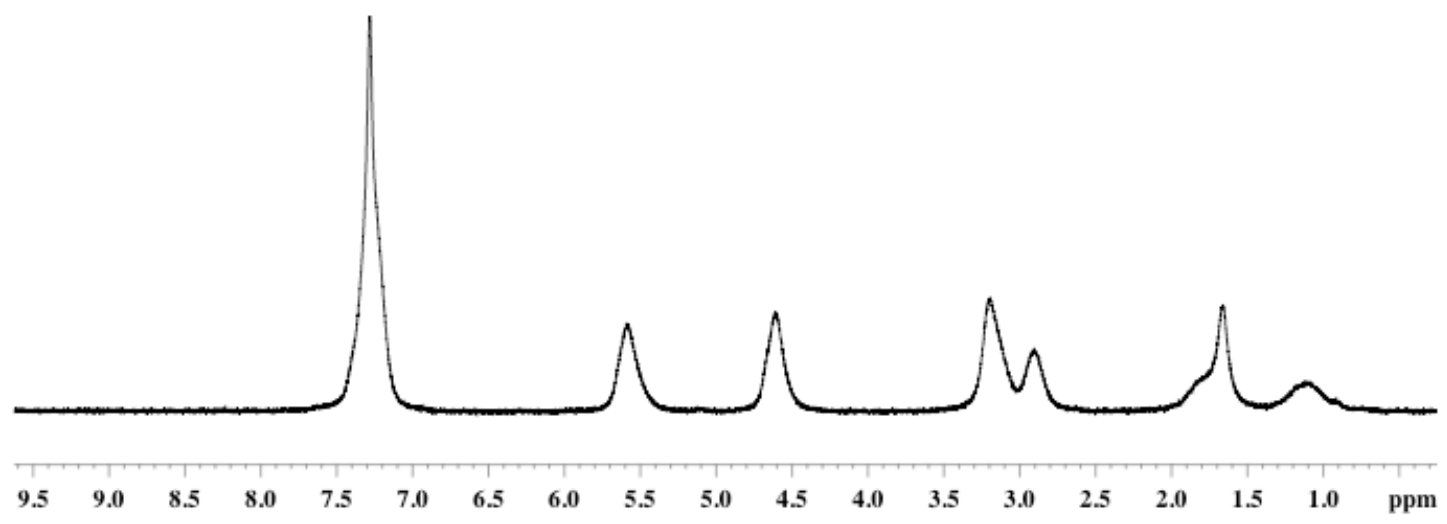

(f) ${ }^{1} \mathrm{H}$ NMR spectrum $\left(300 \mathrm{MHz}, \mathrm{CDCl}_{3}\right)$ of $[\mathbf{5 c}]_{50}$. 


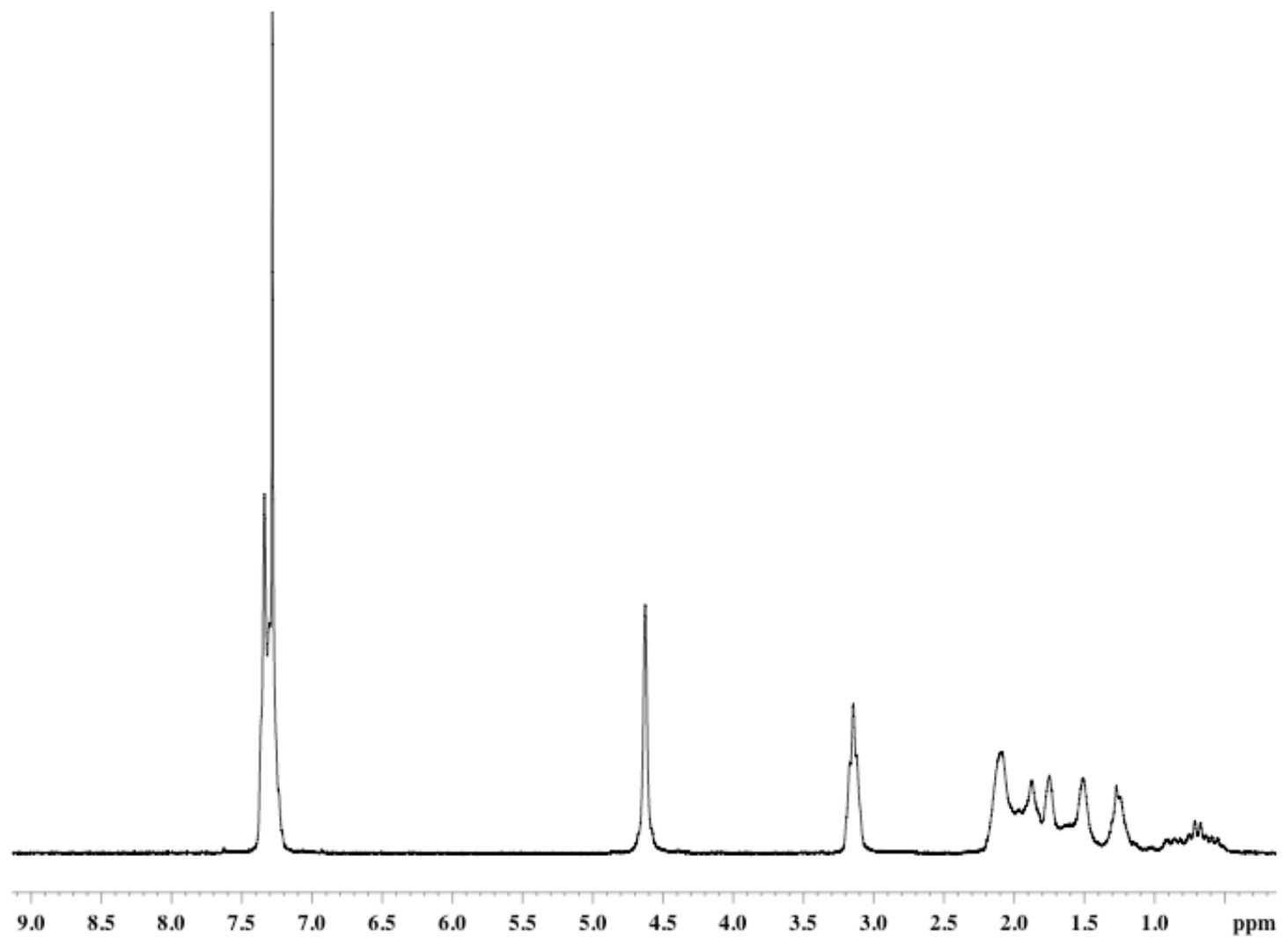

(g) ${ }^{1} \mathrm{H}$ NMR spectrum $\left(300 \mathrm{MHz}, \mathrm{CDCl}_{3}\right)$ of reduced $[\mathbf{5 c}]_{50}$. 


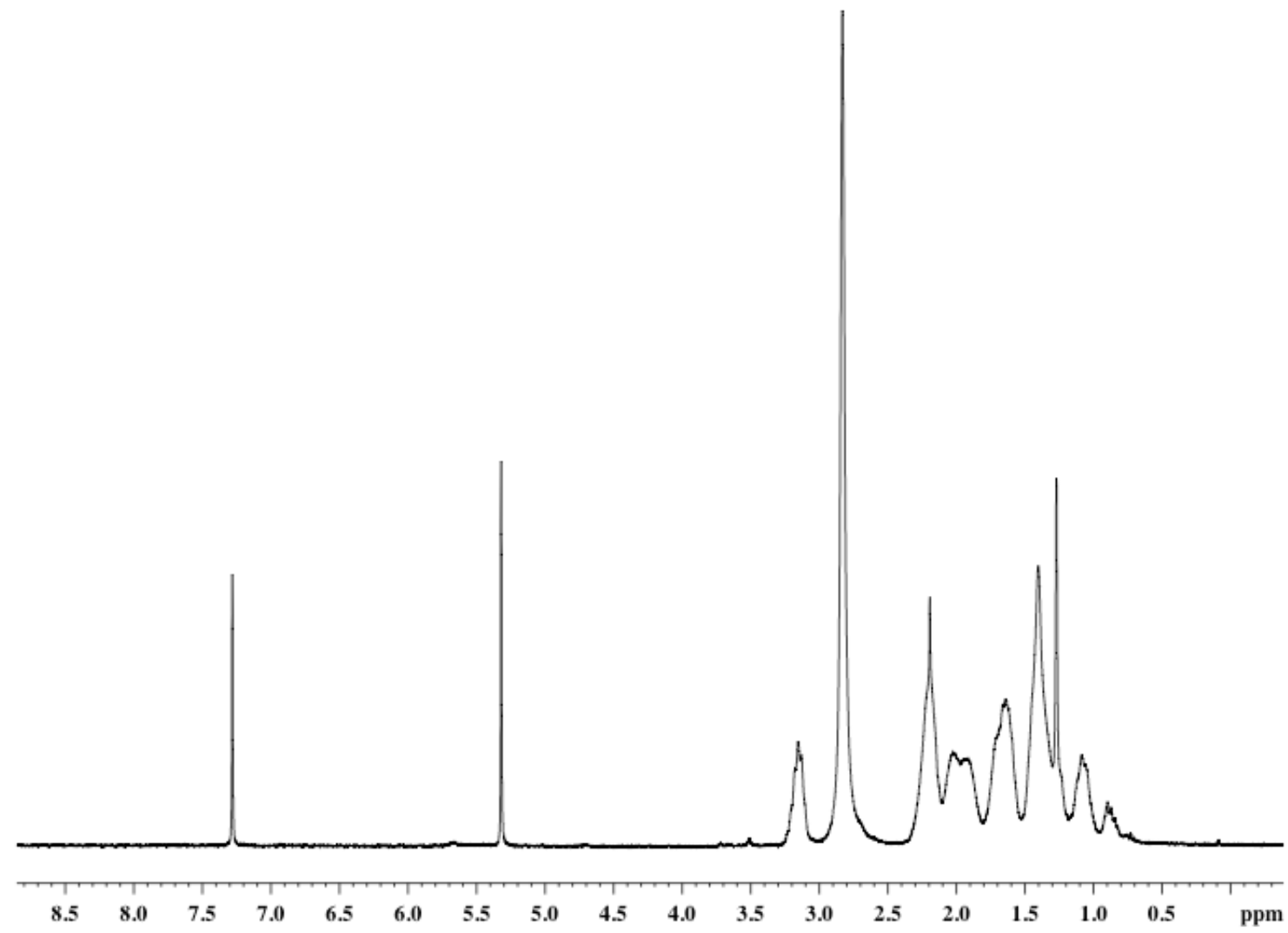

(h) ${ }^{1} \mathrm{H}$ NMR spectrum $\left(300 \mathrm{MHz}, \mathrm{CDCl}_{3}\right)$ of reduced $[\mathbf{5 d}]_{800}$.

\section{References}

(1) Schwab, P.; Grubbs, R. H.; Ziller, J. W. J. Am. Chem. Soc. 1996, 118, 100-110.

(2) Scholl, M.; Ding, S.; Lee, C. W.; Grubbs, R. H. Org. Lett. 1999, 1, 953-956.

(3) Huang, J.; Stevens, E. D.; Nolan, S. P.; Petersen, J. L. J. Am. Chem. Soc. 1999, 121, 26742678.

(4) Sanford, M. S.; Love, J. A.; Grubbs, R. H. Organometallics 2001, 20, 5314-5318.

(5) Conrad, J. C.; Amoroso, D.; Czechura, P.; Yap, G. P. A.; Fogg, D. E. Organometallics 2003, 22, 3634-3636.

(6) Nomura, K.; Schrock, R. R. Macromolecules 1996, 29, 540-545.

(7) Sunaga, T.; Ivin, K. J.; Hofmeister, G. E.; Oskam, J. H.; Schrock, R. R. Macromolecules 1994, 27, 4043-50.

(8) Strong, L. E.; Kiessling, L. L. J. Am. Chem. Soc. 1999, 121, 6193-6196.

(9) Khosravi, E.; Al-Hajaji, A. A. Eur. Pol. J. 1998, 34, 153-157.

(10) Haigh, D. M.; Kenwright, A. M.; Khosravi, E. Tetrahedron 2004, 60, 7217-7224. 